\title{
Implementation of Elliptic Curve Cryptography with Built-In Counter Measures against Side Channel Attacks
}

\author{
Elena Trichina ${ }^{1}$ and Antonio Bellezza ${ }^{2}$ \\ 1 Cryptographic Design Center, Gemplus Technology R \& D \\ Via Pio Emanuelli 1, 00143 Rome, Italy \\ elena.trichina@gemplus.com \\ 2 University of Rome "La Sapienza", Rome, Italy \\ abellezza@tiscalinet.it
}

\begin{abstract}
Many software implementations of public key cryptosystems have been concerned with efficiency. The advent of side channel attacks, such as timing and power analysis attacks, force us to reconsider the strategy of implementation of group arithmetic. This paper presents a study of software counter measures against side channel attacks for elliptic curve cryptosystems.

We introduce two new counter measures. The first is a new implementation technique, namely, homogeneous group operations, which has the property that addition and doubling on elliptic curves cannot be distinguished from side channel analysis. Being inexpensive time-wise, this technique is an alternative to a well-known Montgomery ladder. The second is a non-deterministic method of point exponentiation with precomputations. Although requiring rather large ROM, it provides an effective resistance against high-order power analysis attacks for the price of index re-computations and ROM accesses.

An experimental implementation of NIST-recommended elliptic curves over binary fields with a balanced suite of counter measures built-in in group arithmetic is presented, and the penalty paid is analyzed. The results of the implementation in $\mathrm{C}$ on an AMD Duron $600 \mathrm{MHz}$ running Linux are included in the paper.
\end{abstract}

\section{Introduction}

With the advance of side channel attacks both hard- and software implementations of cryptosystems have to take into account various protection techniques. It has been claimed that all "naive" implementations can succumb to attacks by power analysis. In this paper we do not detail the principles and techniques of different side channel attacks; it had been done elsewhere $17 / 18 / 22|10| 23$. Instead, we survey and systematize different software counter measures against such attacks, including a couple of new techniques, and suggest a balanced approach to their implementation in the context of elliptic curve cryptosystems (ECC) in binary fields. Giving a due to other excellent papers on practical realizations of ECC [29|31|19], we claim that the contribution of our paper is an 
attempt to extend the work in [1] to an implementation in which a balanced suite of counter measures against side channel attacks have been built-in on a group operation level.

An implementation of an elliptic curve cryptosystem involves many choices; not only domain parameters (e.g., underlying finite fields, field representations, particular elliptic curves) and group arithmetic, but also field arithmetic, which, in its turn, includes many different methods and algorithms. It is not surprising therefore that although numerous papers on various aspects of ECC implementations had been written, there are only a handful of those that constitute an extensive and careful study that includes all factors involved in the efficient software implementation, notably 11] and 44. These papers present C implementations on the Pentium $400 \mathrm{MHz}$ workstation of the NIST-recommended elliptic curves over binary and prime fields, respectively.

All published so far complete implementations of ECC have been concerned with efficiency. The advent of side channel attacks force us to reconsider the development cycle. As had been demonstrated in a break-through work by Kocher et al. 1718, for real life implementations an attacker can be able to perform measurements on the device. If the different operations present different characteristics detectable from the outside (like power consumption profile or duration), the sequence of operations can be discovered by the attacker.

In the most widely used cryptographic systems, an exponentiation with a secret exponent is required at some stage. "Exponentiation" in this context is the repeated application of a group operation to the same element. Efficient exponentiation algorithms involve a sequence of squarings, multiplications and possibly divisions (respectively doublings, additions and possibly subtractions in additive notation). Finding out the sequence of operations gives information on the exponent and in some cases uniquely determines it. A detailed systematic description of power analysis attacks on modular exponentiation algorithms can be found in the work of Messerges et al. [22.

Coron [7] generalized DPA attacks to elliptic curve cryptosystems and had shown how to extend DPA to any scalar multiplication algorithm.

Since then, a plethora of papers suggesting various counter measures both, in a general setting and for particular classes of elliptic curves, has been published. We set upon identifying a set of sufficiently general protection techniques that can be employed in a real life scenario. In what follows we survey algorithmic counter measures suggested in the literature, and introduce some original methods. After which an outline of the ECC software implementation in binary fields with a balanced choice of counter measures against side channel attacks is discussed. A description of the experiment with a $\mathrm{C}$ implementation on a $\mathrm{PC}$ with AMD Duron 600 MHZ running Linux, concludes the paper.

\section{Preliminaries and Notation}

Since we are interested in elliptic curves, the notation is additive. Thus the basic operations are doubling, addition and subtraction. We focus on elliptic curves over finite fields of characteristic 2 , which have an affine equation of the form 


$$
y^{2}+x y=x^{3}+a x^{2}+b
$$

for two elements $a$ and $b$ in the base field. A point on the curve is either identified by a couple of affine coordinates $\left(P_{x}, P_{y}\right)$ or is the special point $P_{\infty}$. If $P$ and $Q$ are points on the curve, then an addition $\oplus$ is defined so that $P \oplus Q$ is also a point on the curve and $P_{\infty}$ is the neutral element. Thus one can also define a subtraction $P \ominus Q$. In what follows, notation from [2] is used.

Let $P_{1}=\left(x_{1}, y_{1}\right)$ and $P_{2}=\left(x_{2}, y_{2}\right)$ be points on the curve. Assume $P_{1}, P_{2} \neq$ $P_{\infty}$ and $P_{1} \neq-P_{2}$. The sum $P_{3}=\left(x_{3}, y_{3}\right)=P_{1} \oplus P_{2}$ is computed as follows.

$$
x_{3}=\lambda^{2}+\lambda+x_{1}+x_{2}+a, \quad y_{3}=\left(x_{1}+x_{3}\right) \lambda+x_{3}+y_{1},
$$

where $\lambda=\left(y_{1}+y_{2}\right) /\left(x_{1}+x_{2}\right)$ if $P_{1} \neq P_{2}$, and $\lambda=\left(y_{1}\right) /\left(x_{1}\right)+x_{1}$ if $P_{1}=P_{2}$.

Each point has also a projective expression of the form $\left(P_{X}, P_{Y}, P_{Z}\right)$. If the point is $P_{\infty}$ then its projective coordinates are $(1,1,0)$. Else they are related to the affine coordinates via the transformation rules

$$
\begin{aligned}
\left(P_{x}, P_{y}\right) & \mapsto\left(P_{x}, P_{y}, 1\right) \\
\left(P_{X}, P_{Y}, P_{Z}\right) & \mapsto\left(P_{X} / P_{Z}^{2}, P_{Y} / P_{Z}^{3}\right)
\end{aligned}
$$

A curve then is specified by the equation $Y^{2}+X Y Z=X^{3}+a X^{2} Z^{2}+b Z^{6}$. Doubling formulas $\left(X_{3}, Y_{3}, Z_{3}\right)=2\left(X_{1}, Y_{1}, Z_{1}\right)$ for the projective equation is:

$$
Z_{3}=X_{1}^{2} Z_{1}^{2}, \quad X_{3}=X_{1}^{4}+b Z_{1}^{4}, \quad Y_{3}=b Z_{1}^{4} Z_{3}+X_{3}\left(a Z_{3}+Y_{1}^{2}+b Z_{1}^{4}\right) .
$$

Addition $\left(X_{3}, Y_{3}, Z_{3}\right)=\left(X_{1}, Y_{1}, Z_{1}\right)+\left(X_{2}, Y_{2}, 1\right)$ in mixed coordinates is:

$$
\begin{aligned}
& A=Y_{2} Z_{1}^{2}, B=X_{2} Z_{1}+X_{1}, C=Z_{1} B, D=B^{2}\left(C+a Z_{1}^{2}\right), Z_{3}=C^{2}, E=A C, \\
& X_{3}=A^{2}+D+E, \quad F=X_{3}+X_{2} Z_{3}, \quad G=X_{3}+Y_{2} Z_{3}, \quad Y_{3}=E F+Z_{3} G .
\end{aligned}
$$

ECC is based on the general problem of exponentiation in Abelian groups. Various algorithms for exponentiation in the context of cryptography are presented in [212]. The survey [8] describes fast methods, including those specialized for elliptic curves. Due to a short bit-length of the exponent, and that it is often generated on-the-fly, sophisticated techniques do not show their advantage. However, certain idiosyncrasies of elliptic curves (e.g., subtraction and addition are almost identical), prompt us to consider together with a classical binary method signed and non-adjacent form (NAF) representation of the exponent [21].

Some algorithms described in this paper work with different exponentiation procedures. In order to be able to interchange them, we rely on a routine that transforms an integer into a sequence indicating the order of operations to perform. Possible contents of the resulting sequence elements are $D$ (double), $A$ (add), $S$ (subtract), suitably encoded.

\section{Taxonomy of Software Counter Measures}

Three main techniques developed by Kocher et al., are timing attacks [17], and simple (SPA) and differential power analysis (DPA) attacks [18]. Among them, 
the DPA is the most sophisticated; nevertheless, there are references in the literature when a DPA-protected implementation could be SPA-vulnerable 9 . Moreover, for ECC it seems more difficult to prevent SPA than DPA, as there are good solutions for the latter 7,15,22].

Side channel attacks work because there is a correlation between the physical measurements taken during computations (e.g., power consumption, computing time, EMF radiation, etc.) and the internal state of the processing device, which is itself related to the secret key. While some authors insist on so-called provable resistance 69. to specific classes of attacks, we share an attitude of [5] and state that, unlike in classical cryptology, in a case of side-channel attacks the definition of an adversarial model is not absolute. Thus, rather than having a proof of security, one can have only a strong evidence that the countermeasure resists an attack. Note that the security is assessed at the implementation level.

Three general algorithmic techniques to defy side channel attacks are:

1. Indistinguishability, which basically means that the only strategy for an adversary is to guess at random. It can be partially achieved by eliminating disparity between group operations involved in exponentiation.

2. Random splitting of every bit of the original computation into $k$ shares where each share is equiprobably distributed. Computation then will be carried securely by performing computation only on shares, without reconstructing the original bit 6]9. In practice, an implementation of this technique for ECC amounts to blinding an exponent or/and a base point with some random mask generated for every run.

3. Randomization of the execution sequence which, in order to be successful, must be carried out extensively [6] and, ideally, supported by hardware [27.

In what follows we systematically describe a wide range of counter measures proposed so far for ECC in a general setting.

\subsection{Indistinguishability}

The first requirement to an effective protection against timing and SPA attacks is to ensure that an instruction performed during an execution of a cryptographic algorithm should not be directly dependent on the data being processed.

Double-and-Add Always. Introduced in [7], this counter measure eliminates branch instructions, conditioned by secret data.

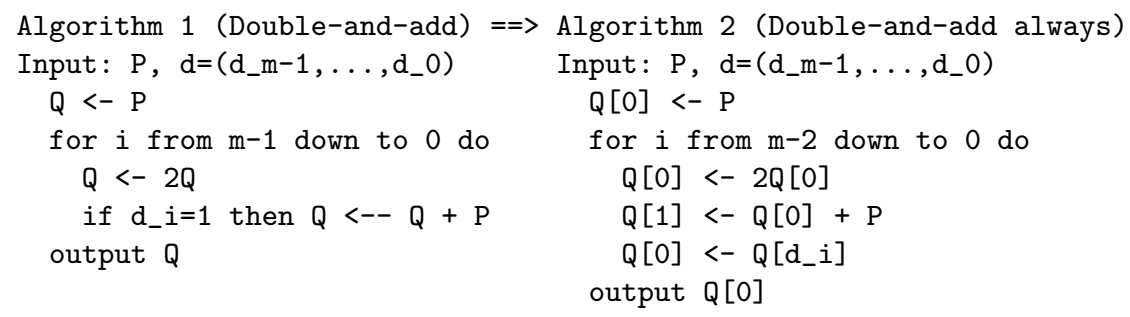


This method increases by $33 \%$ the amount of field operations by necessitating "dummy" computations. As has been shown in [28], it makes it susceptible to a new type of attacks, where an attacker induces any temporary random fault into the ALU during the execution of $Q[1] \leftarrow Q[0]+P$ at iteration $i$. Then, according to whether the final result is incorrect or not, the attacker may deduce if the $d_{i}$ bit of the exponent is 0 or 1 !

The Universal Addition on Elliptic Curves. This method was suggested in [20] for projective coordinates for a subclass of elliptic curves in finite fields of characteristic greater than 3. The approach involves a representation of an elliptic curve as the intersection of two quadric surfaces in $P^{3}$. A non-standard definition of point addition has the advantage that the set of equations holds when two points are equal, thus, naturally eliminating the difference between point addition and doubling. The price to pay is that every point addition requires 16 field multiplications and 3 squarings. Even after various optimizations (that involve the Jacobi representation and a windowing method, and require additional memory), a total increase in computation costs is $70 \%$ in comparison with a standard projective coordinate method.

Independently, a similar approach was developed for Hessian curves in [14, where due to the high symmetry of the Hessian parameterization, the same algorithm can be used for point addition and point doubling. An implementation of point addition requires 12 field multiplications, providing $33 \%$ improvement over Jacobian curves.

Unfortunately, both Jacobian and Hessian parameterizations are not fully general. Brier and Joye later extended the technique in 3] to general Weierstrass elliptic curves and come up with the unified formulae, which requires 7 multiplications, 1 inversion and 3 squarings for affine coordinates and 18 multiplications for projective. This is very expensive in comparison with the method proposed in this paper while providing the same protection.

A Montgomery Ladder. The advantages of Montgomery scalar multiplication 24 in the context of elliptic curves were re-discovered several times 1925 , 13:16]. The Montgomery ladder is based on the binary method and the observation that the $x$-coordinate of the sum of two points whose difference is known, can be computed in terms of only $x$-coordinates of the involved points. Incidentally, it is also the most promising candidate for a "side channel indistinguishable" exponentiation. The Montgomery ladder protected with a "no-branches" technique is given below.

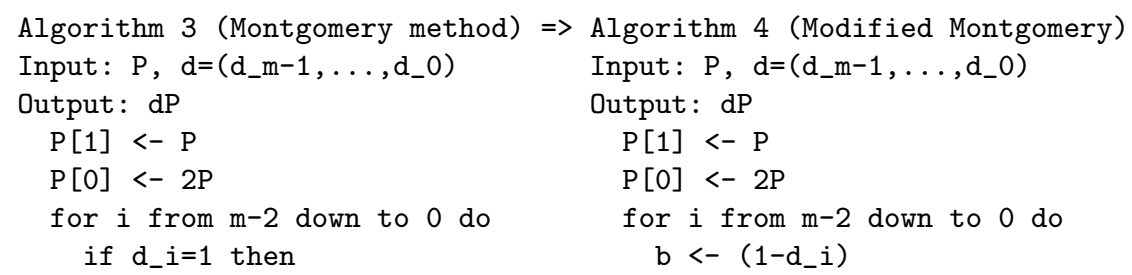




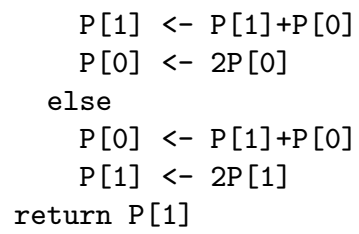

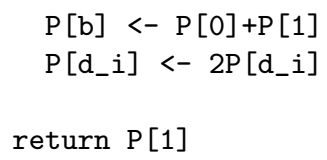

The advantage of this method is perfectly symmetric, memory-efficient computations. The disadvantage is increase in computation costs since both, point addition and point doubling, are computed in each iteration.

An optimization of the Montgomery method based on a new formulae for computing $x$-coordinate for addition of two points, was suggested in [19]. In affine case, a new formulae requires 2 field inversions, 2 multiplications, 2 squarings and 4 additions per iteration; for projective coordinates, it involves only 6 field multiplications, 6 squarings, and 3 additions per iteration. This is comparable cost-wise with our homogeneous group operation method described later, but has an advantage of using less memory, since $y$-coordinate is not used.

Universal Exponentiation Algorithm. Introduced in [5], this is an elegant attempt to design a provably SPA-resistant exponentiation method. Using a representation of the exponent with addition chains, the authors "transfer" the security of the exponentiation method actually implemented in the exponent itself (i.e., in a secret data). The requirement here is that an exponent must be represented as an addition chain in a secure environment. The algorithm works with virtually all exponentiation methods. It reads triplets of values $(\gamma(i): \alpha(i)$ : $\beta(i)$ ) meaning that the content of register $R[\alpha(i)]$ must be multiplied by (added to) the content of register $R[\beta(i)]$ and the result must be written into register $R[\gamma(i)]$. The exponent $d$ then is represented by the register sequence $\Gamma(d)=$ $\{(\gamma(i): \alpha(i), \beta(i))\}_{0 \leq i \leq m-1}$. In order to break the algorithm, an adversary must be able to differentiate among the triplets and to recover all their values. The advantage of this method is that it introduces only a small amount of extra computations; the disadvantage is large memory usage.

\subsection{Splitting Variables}

In practice, the general encoding proposed in 69 amounts to blinding an exponent and a base point with some random mask to prevent $D P A$ attacks.

Blinding an Exponent. Randomization of the exponent is inexpensive and useful technique. It comes in few flavors.

An additive mask is usually used in the ECC context [7]. It consists in adding a multiple of the element order to the exponent. If $N$ is the group order, then $(k N) P=0$ for any integer $k$ and for any element $P$ in the group. To compute $d P$, where $d$ is the secret exponent and $P$ is a base point, one first blinds $d$ 
with $k N$, where $k$ is a (small) random number generated for every run, and $N$ is the group order. Hence, $Q=(d+k N) P=d P+(k N) P=d P$. As $d$ usually is the size of the group order, choosing a $t$-bit integer $k$ increases the size of the exponent by about $t$ bits.

An exponent splitting [5] is a variant of this technique, where $d$ is represented as a sum of a random $k$ and $d-k$, and exponentiation is carried out in two steps: $R \rightarrow k P ; \quad Q \rightarrow(d-k) R$. The values of both, $k$ and $d-k$ are required to recover the value of $d$, which makes it less attractive than the method above.

A multiplicative mask is a multiplicative analogue of this idea. Let $N$ be the group order. If $k$ is a unit in the multiplicative group $(\mathbf{Z} / \mathbf{Z} N, \times)$ and $k^{-1}$ its inverse, then $d=k\left(k^{-1} d\right)(\bmod N)$ for any exponent $d$. This means that calling $d^{\prime}:=k^{-1} d(\bmod N)$, for any group element $P$ one has the following equivalent exponentiations:
(1) $P \stackrel{d}{\mapsto} d P$
(3) $P \stackrel{d^{\prime}}{\mapsto} d^{\prime} P \stackrel{k}{\mapsto} k\left(d^{\prime} P\right)$
(2) $P \stackrel{k}{\mapsto} k P \stackrel{d^{\prime}}{\mapsto} d^{\prime}(k P)$
(4) $P \stackrel{k d^{\prime}}{\mapsto}\left(k d^{\prime}\right) P$

Sequence (1) is a straightforward exponentiation; sequence (4) is a sub-case of the additive blinding technique. Sequences (2) and (3) are performed in two phases. We focus on (2). The overhead is given by the exponentiation with exponent $k$, since both $d$ and $d^{\prime}$ are on average the size of $N$. To keep overhead low, one can choose $k$ to yield a fast exponentiation, for instance by choosing it randomly among the elements of $(\mathbf{Z} / \mathbf{Z} N)^{*}$ of at most $t$ bits, with a small $t$. As in the additive blinding, one can trade-off the degree of randomization (and thus the security) for speed.

If sequence (2) is used, the element fed to the second phase is not controlled by an attacker. The first phase, on the other hand, doesn't leak any information whatsoever on the secret exponent, depending uniquely on the input element and the random integer $k$. Thus one gets at the same time additional bonuses of a change in the sequence of point operations and of randomization of the input point. Cost-wise, the method is comparable with the additive mask, although precomputations are somewhat slower.

Blinding a Base Point. Blinding a base point is necessary if we assume that an attacker knows how points are represented in memory during computations [7. An accepted technique to mask the input by applying some bijective function for which it is easy to compute the final correction.

Adding to the input a "perturbation point" [7] involves storing a couple of "random" points $(R,-d R)$ and updating it at each run by doubling both elements. Exponentiation of an input $P$ with an exponent $d$ is performed in three steps: (1) $P^{\prime} \leftarrow P+R, \quad(2) Q^{\prime} \leftarrow d P^{\prime}=d P+d R, \quad(3) Q \leftarrow Q^{\prime}+(-d R)=d P$. 
As the argument $P+R$ of the exponentiation phase does not depend exclusively on the input, it is not possible to choose points with particular properties. However, if $d$ changes at each run, computing $-d R$ for final correction doubles group operations, and must be protected as well.

Randomizing projective coordinates of a point [7] is achieved almost for free. Projective coordinates of a point is not unique because $(X, Y, Z)=$ $(k X, k Y, k Z)$ for every $k \neq 0$ in the finite field. Hence, before each new execution of $d P$, the projective coordinates of $P$ can be randomized with a random $k$. It makes it impossible for an attacker to predict any specific bit of the binary representation of $P$, thus rending a DPA attack infeasible.

\subsection{Randomization of the Execution Sequence}

Non-deterministic execution of the sequence of instructions, although does not provide perfect protection against statistical techniques, increases the number of measurements.

Randomized Addition/Subtraction chain. [26] exploits the fact that $d P$ can be computed with different execution sequences; for instance, for $9 P: P \rightarrow$ $2 P \rightarrow 4 P \rightarrow 8 P \rightarrow 9 P$ or $P \rightarrow 2 P \rightarrow 4 P \rightarrow 5 P \rightarrow 10 P \rightarrow 9 P$. Randomization can be achieved by inserting a random decision that can rearrange a sequence of additions, subtractions and doubling in a signed binary algorithm. The idea is to randomly substitute a long chain of 1's (which correspond to "double-andadd") in the signed binary representation of the exponent by a block of zeros ("double") followed by -1 (subtract). Similar substitution is applied to isolated 0 's inside a block of 1's. The algorithm needs some $9 \%$ more additions. The authors claim that it makes DPA attacks really difficult.

\section{Homogeneous Group Operations}

In the choice of doubling and addition algorithms for elliptic curves, it is often advisable to work in projective coordinates, which allows us to avoid costly inversions in the field. For elliptic curves over fields of characteristic 2, complexities for doubling performed on projective coordinates and addition and subtraction on mixed affine-projective coordinates with projective result, are: Here Mult stands for multiplication, Squar for squaring, and Add for addition.

The problem we address here is the relevant computational difference between addition/subtraction and doubling, which in implementation reflects in differences detectable by an attacker. Observing that the complexity for point doubling is about half of the complexity for addition/subtraction, one can think of splitting the complex subroutines into two parts, each one approximately equivalent to a point doubling. This is actually possible, as we show below.

When choosing an elliptic curve, setting $a=1$ makes the total number of products in addition and subtraction exactly twice the number for doubling, 
Table 1. Complexities of group operations in projective coordinates

\begin{tabular}{|l||ccc||ccc|}
\hline \multicolumn{1}{|c||}{} & \multicolumn{3}{c|}{ General case } & \multicolumn{3}{c|}{ Case $a=1$} \\
\hline \hline Curve operation & Mult & Squar & Add & Mult & Squar & Add \\
\hline Doubling & 5 & 5 & 4 & 5 & 5 & 4 \\
Addition & 11 & 3 & 7 & 10 & 3 & 7 \\
Subtraction & 11 & 3 & 8 & 10 & 3 & 8 \\
\hline
\end{tabular}

thus avoiding dummy operations. We present atomic units of computations in the case $a=1$. A general case slightly differs in the addition/subtraction part, while the doubling subroutine is the same.

Before proceeding, we observe that computing the subtraction $P \ominus Q$ of two points corresponds to computing $P \oplus(-Q)$. If $Q$ has affine coordinates $\left(Q_{x}, Q_{y}\right)$, then $-Q$ has affine coordinates $\left(Q_{x}, Q_{y}+Q_{x}\right)$. Thus, the algorithms for addition and subtraction only differ by a field addition, and we will consider them at the same time. Furthermore, if the affine point to be subtracted is the same throughout a loop, one can compute $Q_{x}+Q_{y}$ once. Here are the subroutines.

Doubling. The input is given by the coordinates $\left(A_{X}, A_{Y}, A_{Z}\right)$ of the point.

$$
\begin{array}{lll}
\lambda_{1} \longleftarrow A_{Z}^{2} & \lambda_{6} \longleftarrow \widetilde{b} \lambda_{1} & \lambda_{11} \longleftarrow \lambda_{7}^{4} \\
\lambda_{2} \longleftarrow A_{X} \lambda_{1} & \lambda_{7} \longleftarrow A_{X}+\lambda_{6} & \lambda_{12} \longleftarrow \lambda_{10} \lambda_{11} \\
\lambda_{3} \longleftarrow A_{Y} A_{Z} & \lambda_{8} \longleftarrow \lambda_{4}^{2} & \lambda_{13} \longleftarrow \lambda_{9}+\lambda_{12} \\
\lambda_{4} \longleftarrow A_{X}^{2} & \lambda_{9} \longleftarrow \lambda_{8} \lambda_{2} & \\
\lambda_{5} \longleftarrow \lambda_{2}+\lambda_{4} & \lambda_{10} \longleftarrow \lambda_{5}+\lambda_{3} &
\end{array}
$$

Here $\widetilde{b}$ is such that $\widetilde{b^{4}}=b$. It can be computed once and for all when the curve is set. The output point has projective coordinates $\left(\lambda_{11}, \lambda_{13}, \lambda_{2}\right)$. The sequence of operations is SMMSAMASMASSMA.

Addition/Subtraction - Phase I (Case $\boldsymbol{a}=\mathbf{1}$ ). The input is given by projective coordinates $\left(A_{X}, A_{Y}, A_{Z}\right)$ and affine coordinates $\left(B_{x}, B_{y}\right)$.

$$
\begin{aligned}
& \mu_{1} \longleftarrow \lambda_{7}^{2} \quad \lambda_{4} \longleftarrow B_{y} \quad \text { (addition) } \quad \lambda_{7} \longleftarrow A_{Z} \lambda_{6} \\
& \lambda_{1} \longleftarrow A_{Z}^{2} \quad \text { or } \quad \lambda_{8} \longleftarrow A_{Y}+\lambda_{5} \\
& \lambda_{2} \longleftarrow \lambda_{1} B_{x} \quad \lambda_{4} \longleftarrow B_{y}+B_{x} \quad \text { (subtraction) } \quad \lambda_{9} \longleftarrow \lambda_{8} B_{x} \\
& \lambda_{3} \longleftarrow \lambda_{1} A_{Z} \quad \lambda_{5} \longleftarrow \lambda_{4} \lambda_{3} \quad \lambda_{11} \longleftarrow \lambda_{7}+\lambda_{8} \\
& \lambda_{6} \longleftarrow A_{X}+\lambda_{2}
\end{aligned}
$$

The sequence of operations is SMMAMAMAMA. 
Addition/Subtraction - Phase II (Case $\boldsymbol{a}=\mathbf{1}$ ). This subroutine is executed right after the previous one, of which it uses some of the partial results.

$$
\begin{array}{lll}
\mu_{1} \longleftarrow \lambda_{7}^{2} & \mu_{5} \longleftarrow \mu_{1}+\mu_{3} & \mu_{9} \longleftarrow \lambda_{9}+\mu_{2} \\
\mu_{2} \longleftarrow \lambda_{7} \lambda_{4} & \mu_{6} \longleftarrow \lambda_{6} \mu_{4} & \mu_{10} \longleftarrow \mu_{9} \mu_{1} \\
\mu_{3} \longleftarrow \lambda_{8} \lambda_{11} & \mu_{7} \longleftarrow \mu_{5}+\mu_{6} & \mu_{11} \longleftarrow \mu_{8}+\mu_{10} \\
\mu_{4} \longleftarrow \lambda_{6}^{2} & \mu_{8} \longleftarrow \mu_{7} \lambda_{11} &
\end{array}
$$

The output point has projective coordinates $\left(\mu_{7}, \mu_{11}, \lambda_{7}\right)$. The sequence of operations is SMMSAMAMAMA.

The shortest operation sequence (including a couple of dummy operations) for all three subroutines is $S M M S A M A S M A S S M A$.

Table 2. Complexities of homogeneous vs normal group operations

\begin{tabular}{|l||ccc||ccc|}
\hline \multicolumn{1}{|c||}{} & \multicolumn{3}{c|}{ General case } & \multicolumn{3}{c|}{ Case $a=1$} \\
\hline \hline Curve operation & Mult Squar Add & Mult Squar & Add \\
\hline Doubling (old) & 5 & 5 & 4 & 5 & 5 & 4 \\
Doubling (new) & 6 & 5 & 4 & 5 & 5 & 4 \\
\hline Addition (old) & 11 & 3 & 7 & 10 & 3 & 7 \\
Addition (new) & 12 & 10 & 8 & 10 & 10 & 8 \\
\hline Subtraction (old) & 11 & 3 & 8 & 10 & 3 & 8 \\
Subtraction (new) & 12 & 10 & 8 & 10 & 10 & 8 \\
\hline
\end{tabular}

Table 2 gives the complexities for various options. Observe that squarings and additions in the field of characteristic 2 are very cheap. Thus we are wasting little more than one field multiplication per curve operation in the general case, and just a few additions and squarings in the case $a=1$.

\section{An Exponentiation Algorithm Using Homogeneous Point Operations.}

The classical algorithms can be used to implement an exponentiation that shows to the outside world a sequence of homogeneous rounds. The subroutines for doubling, addition-phase I, subtraction-phase I and addition/subtraction-phase II have been implemented in C. In order to achieve homogeneity, they are invoked with the same kind of parameters, i.e. with 5 field elements representing the projective and affine coordinates of two curve points. The result is a point in projective coordinates, i.e. a triplet of field elements.

Phase I of addition and subtraction returns the first input point (the one in projective coordinates). The subroutines are called $F_{i}$ with $i$ being 0 for doubling, 1 for addition/I, 2 for subtraction/I and 3 for addition-subtraction/II. $F_{1}$ and $F_{2}$ also have side-effects, namely they store in memory partial results which are used by $F_{3}$. We suppose that at the precomputation step, the exponent is transformed in a secure environment into a sequence $\mathrm{b}[1], \ldots, \mathrm{b}[\mathrm{d}]$ corresponding to procedure calls. 
Algorithm 5 (Exponentiation with homogeneous group operations)

Input: point $\mathrm{P}=\left(\mathrm{P}_{-} \mathrm{x}, \mathrm{P}_{-} \mathrm{y}\right)$ in affine coordinates, sequence $b[1], \ldots, b[d]$ of atomic unit calls for exponent $d$

Output: point $\mathrm{dP}$ in affine coordinates

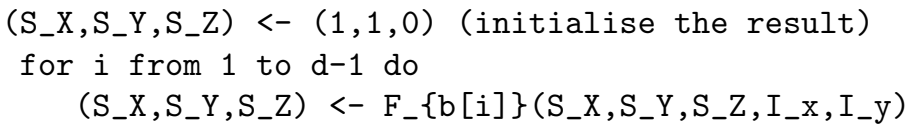

Final conversion

S_X $<-S_{-} X / S_{-} Z \sim 2$

S_y $<-S_{-} Y / S_{-} Z \wedge 3$

Return the point with affine coordinates (S_x,S_y)

\section{Non-deterministic Right-to-Left Method with Precomputations}

Non-deterministic right-to-left method with precomputations had been actually implemented in our experiment. It is very efficient but requires a lot of ROM.

An algorithm is based on the observation that $d P=d_{m-1} 2^{m-1} P+\ldots+$ $d_{1} 2 P+d_{0} P$, where $\left(d_{m-1}, \ldots, d_{1}, d_{0}\right)$ is a binary representation of the exponent $d, d_{0}$ being the LSB, and $d_{m-1}$ the MSB of $d$. Thus, if the multiples of point $P$ are precomputed in advance, it does not matter in which order the bits of the exponent $d$ are scanned as long as one can associate the bit $d_{i}$ with the precomputed multiple $2^{i} P$. Here is our solution.

Algorithm 6 (Non-deterministic right-to-left with precomputations)

Input: array $A=\left[2^{\wedge}\{m-1\} P, \ldots, 2 P, P\right]$ of precomputed multiples of $P$

$d=\left(d_{-}\{m-1\}, \ldots, d_{-} 1, d_{-} 0\right)$ bits of an exponent

Output: dP

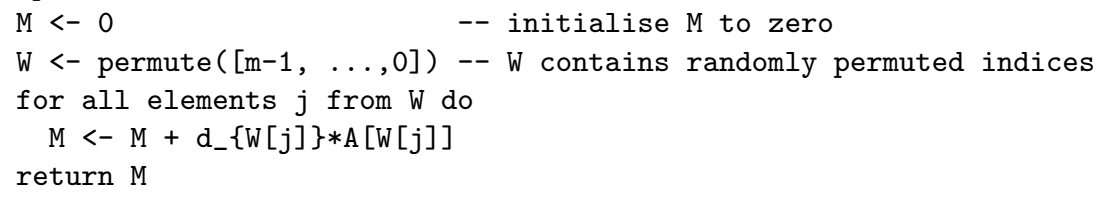

Instead of permutation, one can just rotate $A$ and $d$ simultaneously $j$ positions to the left or to the right; $0 \leq j \leq m-1$ is a random updated at each run. To enhance the randomization, the method of random splitting and rotating can be applied recursively to the sub-arrays of indices. This method is beneficial for protocols where the base point $P$ does not change often. However, it requires additional ROM to store precomputed multiples of $P$. Considering that the size of field elements for the ECC is around 200 bits, the total amount of memory in question is $25 \times \log N$ bytes, where $N$ is a group order.

\section{A Point of a Small Order as a Perturbation Point}

A point of a small order on the curve as a perturbation point is a variation on the idea of blinding a base point. Elliptic curves of use for cryptographic 
purposes have a group of points with order a big prime $N$ times a cofactor $h$, where $h$ is usually small. We suggest choosing as the perturbation point $R$ a point of order $h$. We suppose the cofactor not to be too small (a cofactor of 2 would be no good) but such that its bit-length $l_{h}$ is not bigger than, say, $1 / 5$ of bit-length $l_{N}$ of $N$.

This presents some disadvantages but allows on-the-fly computation of the point and the correction at each run with much fewer group operations. To be useful, a cofactor $h$ has to be at least 4. Although 1] contains an example with the bit-length $l_{h}=16$ and $l_{N}=161$, the trend to have a very small cofactor weakens our method.

We compute a random point of order $h$ as the multiple of $Q$ by a random factor $r$ between 0 and $h-1$. The final correction to apply is $T:=-d r Q$. We can actually compute it as $(-d r \bmod h) Q$. The factor is again between 0 and $h-1$. As the bit-length of $h$ is considerably less than the bit-length of $N$, the exponentiations to get $R$ and $T$ are faster than the main exponentiation and require some $l_{h}$ additions. The computation of $r Q$ and $(-d r \bmod h) Q$ must be masked. As $r$ changes after each run, one has to take care mostly of single-run analysis.

One can speed up and simultaneously mask computations of $r Q$ and $(-d r$ $\bmod h) Q$ by storing $2 Q, 4 Q, \ldots, 2^{l_{h}-1} Q$ and using fast right-to-left binary algorithm in a non-deterministic manner, as described in the previous chapter. This technique had actually been implemented.

\section{Computer Experiments}

We implemented some of the ideas explained in the previous chapters. For finite fields, we implemented in $\mathrm{C}$ basic operations from scratch following [11].

Field Arithmetics. As polynomial basis, we followed [11] and [1, choosing the following irreducible polynomials for the fields in our experiments.

$$
\begin{aligned}
& \mathbf{F}_{2^{163}}=\mathbf{F}_{2}[x] /\left(x^{163}+x^{7}+x^{6}+x^{3}+1\right) \\
& \mathbf{F}_{2^{163}}^{\prime}=\mathbf{F}_{2}[x] /\left(x^{163}+x^{8}+x^{2}+x+1\right) \\
& \mathbf{F}_{2^{233}}=\mathbf{F}_{2}[x] /\left(x^{233}+x^{74}+1\right) \\
& \mathbf{F}_{2^{283}}=\mathbf{F}_{2}[x] /\left(x^{283}+x^{12}+x^{7}+x^{5}+1\right)
\end{aligned}
$$

For multiplication right to left and left to right comb methods, plus a base 16 left to right comb method were implemented. As the inversion algorithm we chose the extended Euclidean algorithm. Sample timings taken on an AMD Duron $600 \mathrm{MHz}$ running Linux for selected fields are given in Table 3.

Exponentiation on Elliptic Curves. We implemented both binary and signed NAF exponentiation for elliptic curves over $F_{2^{163}}$, choosing a curve $E_{1}$ with $a=1$ and a general curve $E_{2}$ with the parameters specified below. 
Table 3. Timing for field operations in $\mu$ s for various fields

\begin{tabular}{|l||cccc|}
\hline \hline Operation & $\mathbf{F}_{2^{163}}$ & $\mathbf{F}_{2^{163}}^{\prime}$ & $\mathbf{F}_{2^{233}}$ & $\mathbf{F}_{2^{283}}$ \\
\hline \hline Addition & 0.16 & 0.16 & 0.17 & 0.16 \\
\hline Squaring & 0.34 & 0.37 & 0.38 & 0.53 \\
\hline Product: RL comb & 7.00 & 7.13 & 9.86 & 18.76 \\
Product: LR comb & 10.04 & 10.10 & 14.93 & 18.72 \\
Product: Base 16 LR comb & 2.78 & 2.80 & 3.61 & 4.59 \\
\hline Inversion & 36.02 & 36.05 & 60.50 & 77.97 \\
\hline
\end{tabular}

Point operations were performed using the standard sequence of operations and the homogeneous versions. Table 4 summarizes the penalty for a side-channel protection. To get the following timings, the generating points were multiplied by a random exponent both with standard and homogeneous operations. For $E_{1}$ the fact that $a=1$ was taken into account in both versions.

As for a real-life scenario, a suite of built-in counter measures included:

- indistinguishability via homogeneous operations (3-17\% overhead);

- a multiplicative exponent masking, where the first step (multiplication with a small random $k$ ) had been implemented via a non-deterministic right-toleft binary method with precomputations, providing an additional benefit of blinding a base point in a process ( $15 \%$ overhead).

The total costs amounted to 2133 field multiplications, 1773 squarings, 1416 additions and 3 inversions, while a non-protected version would have had 1683 multiplications, 1121 squarings, 1200 additions and 1 inversion. In other words, some $30 \%$ increase in computation time constitutes the total penalty.

\begin{tabular}{|c|c|}
\hline & Elliptic curve parameters \\
\hline$\overline{\bar{E} E_{1}}$ & \\
\hline $\mathrm{F}$ & $\mathbf{F}_{2}[x] /\left(x^{163}+x^{7}+x^{6}+x^{3}+1\right)$ \\
\hline $\mathrm{a}$ & - \\
\hline $\mathrm{b}$ & 02 0A601907 B8C953CA 1481EB10 512F7874 4A3205FD \\
\hline & $040000000000000000000292 \mathrm{FE}$ 77E70C12 A4234C33 \\
\hline & 02 \\
\hline & 03 FB02D922 0A5E7980 D9C7C192 AFC7EDC4 19B261E4 \\
\hline & 05 F8692B70 5F82AAF2 7E41D4D3 82D9E359 98979F99 \\
\hline$E_{2}$ & \\
\hline $\mathrm{F}$ & $\mathbf{F}_{2}[x] /\left(x^{163}+x^{8}+x^{2}+x+1\right)$ \\
\hline $\mathrm{a}$ & 07 2546B543 5234A422 E0789675 F432C894 35DE5242 \\
\hline $\mathrm{b}$ & 00 C9517D06 D5240D3C FF38C74B 20B6CD4D 6F9DD4D9 \\
\hline & $040000000000000000000292 \mathrm{FE}$ 77E70C12 A4234C33 \\
\hline & 02 \\
\hline & 07 AF699895 46103D79 329FCC3D 74880F33 BBE803CB \\
\hline & 06 434AB98E 1F769093 2FA04BCA 9ED0479D 4B5FC954 \\
\hline
\end{tabular}




\section{Conclusion}

From our experiment it follows that the cost of counter measures against side channel attacks need not to be prohibitively high. With a choice of well-balanced suite of complementary counter measures the total overhead can be as low as $30 \%$. We want to emphasize that the objective of the experiment was an

Table 4. Exponentiation timings in $\mu \mathrm{s}$

\begin{tabular}{|lll||lll|}
\hline \hline \multicolumn{3}{|c|}{ binary NAF } & \multicolumn{3}{c|}{ binary NAF } \\
\hline$E_{1}$ & & & $E_{2}$ & & \\
\hline standard & 5355 & 4699 & standard & 5642 & 4932 \\
homogeneous & 5512 & 4847 & homogeneous & 6581 & 5770 \\
overhead & $2.9 \%$ & $3.1 \%$ & overhead & $16.6 \%$ & $17.0 \%$ \\
\hline \hline
\end{tabular}

"average-case" analysis, rather than finding the fastest or the most secure solution. This explains, for example, the choice of the field $G F\left(2^{n}\right)$ and the field representation. It is well-known that operations in $G F\left(2^{n}\right)$ can be efficiently implemented in both, hardware and software, and that, although the normal basis representation offers a very fast squaring, the polynomial representation is more widely used.

The choice of projective coordinates for ECC allows us to use homogeneous group operations, thus achieving SPD resistance with only $3-17 \%$ overhead. One can argue that using projective coordinates is a good idea only in prime fields, but not in $G F\left(2^{n}\right)$ [30]. However, the choice of coordinates must take into account not only performance, but also security of the implementation. Performance-wise, it makes sense to use projective coordinates when the ratio inversion/ multiplication is greater than 3. Since this ratio depends very much on the chosen multiplication algorithm (see the table in the previous section), the choice of coordinates cannot be made solely on the criteria of the field.

Projective coordinates offer actually two benefits when considering side channel attack resistance. They can be used to defy DPA attacks by randomizing coordinates as in [7] as well as SPA and timing attacks using our homogeneous group operations method.

The experiment made us also wiser. If we were to give an advise on an allround suite of counter measures against side channel attacks, including timing, SPA, DPA, and fault attacks, we would recommend the following.

- Blinding the base point by randomizing projective coordinates.

- Randomization of the exponent can be done using both, additive or multiplicative masks; the latter has an additional benefit of randomization of the execution sequence, and partially, of a base point.

- Use the securized Montgomery ladder with optimized group operations (on $x$-coordinates only) as suggested in [19. The overhead is comparable with the one achieved in our experiment, but the algorithm is naturally more regular and thus, more secure. 


\section{References}

1. ANSI X9.62: The elliptic curve digital signature algorithm (ACDSA), draft, July 1997.

2. I. Blake, G. Seroussi, N. Smart. Elliptic Curves in Cryptography. Cambridge University Press (1999).

3. Brier, E., Joye,: Weierstrass elliptic curves and side-channel attacks. Proc. Public Key Cryptography (PKC 2002), LNCS 2274 (2002) 335-345

4. Brown, M., Hankerson, D., Lopez, J., Menezes, A.: Software implementation of the NIST elliptic curves over prime fields. Proc. CT-RSA 2001, LNCS 2020 (2001) 250-265

5. Clavier, C., Joye, M.: Universal exponentiation algorithm: A first step towards provable SPA-resistance. Proc. Cryptographic Hardware and Embedded Systems (CHES 2001), LNCS 2162 (2001) 300-308

6. Chari, S., Jutla, C., Rao, J., Rohatgi, P.: Towards sound approaches to counteract power-analysis attacks. Proc. Advances in Cryptology - Crypto'99, LNCS 1666 (1999) 398-412

7. Coron, J.-S.: Resistance against differential power analysis attacks for elliptic curve cryptosystems. Proc. Cryptographic Hardware and Embedded Systems (CHES'99), LNCS 1717 (1999) 292-302

8. Gordon, D. M.: A survey of fast exponentiation methods. J. Algorithms 27 (1998) 129-146

9. Goubin, L., Patarin, J.: DES and differential power analysis. Proc. Cryptographic Hardware and Embedded Systems (CHES'99), LNCS 1717 (1999) 158-172

10. Hasan, M. A.: Power analysis attacks and algorithmic approaches to their countermeasures for Koblitz cryptosystems. Proc. Cryptographic Hardware and Embedded Systems (CHES 2000), LNCS 1965 (2000) 93-108

11. Hankerson, D., Hernandez, J. L., Menezes, A.: Software implementation of elliptic curve cryptography over binary fields. Proc. Cryptographic Hardware and Embedded Systems (CHES 2000), LNCS 1965 (2000)

12. IEEE Std 1363-2000. IEEE Standard Specification for Public-Key Cryptography. IEEE Computer Society, August 29, 2000

13. Izu, T., Takagi, T.: A fast parallel elliptic curve multiplication resistant against side channel attacks. Proc. Public Key Cryptography (PKC 2002), LNCS 2274 (2002) 280-296

14. Joye, M., Quisquater, J-J.,: Hessian elliptic curves and side-channel attacks. Proc. Cryptographic Hardware and Embedded Systems (CHES 2001), LNCS 2162 (2001) 402-410

15. Joye, M., Tymen, Ch.: Protection against Differential Power Analysis for elliptic curve cryptography - An algebraic approach. Proc. Cryptographic Hardware and Embedded Systems (CHES 2001), LNCS 2162 (2001) 377-390

16. Joye, M., Yen, S-M.: The Montgomery powering ladder. In these proceedings.

17. Kocher, P.: Timing attacks on implementations of Diffie-Hellmann, RSA, DSS, and other systems. Proc. Advances in Cryptology - Crypto'96, LNCS 1109 (1996) 104-113

18. Kocher, P., Jaffe, J., Jun, B.: Differential power analysis. Proc. Advances in Cryptology - Crypto'99, LNCS 1666 (1999) 388-397

19. Lopez, J., Dahab, R.: Fast multiplication on elliptic curves over $G F\left(2^{n}\right)$ without precomputations. Proc. Cryptographic Hardware and Embedded Systems (CHES'99), LNCS 1717 (1999) 316-327 
20. Liardet, P.-Y., Smart, N. P.: Preventing SPA/DPA in ECC systems using the Jacobi form. Proc. Cryptographic Hardware and Embedded Systems (CHES 2001), LNCS 2162 (2001) 391-401

21. Menezes, A. J., van Oorschot, P. C., Vanstone, S. A.: Handbook in Applied Cryptography. CRC Press, 1997

22. Messerges, T. S., Dabbish, E. A., Sloan, R. H.: Power analysis attacks on modular exponentiation in smartcards. Proc. Cryptographic Hardware and Embedded Systems (CHES'99), LNCS 1717 (1999)

23. Messerges, T. S., Dabbish, E. A., Sloan, R. H.: Examining smart-card security under the threat of power analysis attacks. IEEE Transactions on Computers, $\mathbf{5 1}$ (5) (2002) 541-552

24. Montgomery, P.: Speeding the Pollard and elliptic curve methods of factorization. Mathematics of Computations, 48 (1987) 243-264

25. Okeya, K., Sakaurai, K.: Power analysis breaks Elliptic Curve cryptosystems even secure against the timing attacks. Proc. INDOCRYPT 2000, LNCS 1977 (2000) $178-190$

26. Oswald, E., Aigner, M.: Randomized addition-subtraction chains as a countermeasure against power attacks. Proc. Cryptographic Hardware and Embedded Systems (CHES 2001), LNCS 2162 (2001) 39-50

27. May, D., Muller, H.L., Smart, N.P.: Non-deterministic processors. Proc. Cryptographic Hardware and Embedded Systems (CHES 2001), LNCS 2162 (2001) 28-38

28. Yen, S-M., Kim, S-J., Lim, S-G., Moon, S-J: A counter-measure against one physical cryptanalysis may benefit another attack. Proc. Information Security and Cryptology (ICISC 2001), LNCS 2288 (2002) 414-427

29. De Win,. E., Bosselaers, A., Vandenberghe, S., De Gersem, P., Vandewalle, J.: A fast software implementation for arithmetic operations in $G F\left(2^{n}\right)$. Proc. Asiacrypt '96, LNCS 1163 (1996) 65-76

30. De Win,. E., Mister, S., Preneel, B., Wiener M.: On the performance of signature schemes based on elliptic curves. Proc. An Algorithmic Number Theory Symposium, LNCS 1423 (1998)

31. Woodbury, A., Bailey, D., Paar, Ch.: Elliptic curve cryptography on smart cards without coprocessors. Proc. The Forth Smart Card Research and Advanced Applications Conf., September 20-22, 2000 Bristol, UK 